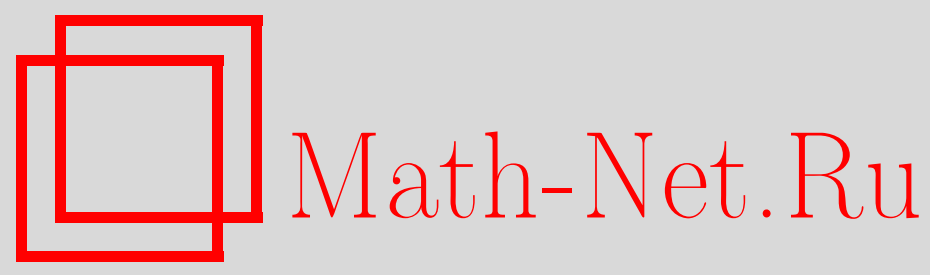

K. Е. Бауман, Оценка снизу квадратно-линейного отношения для правильных кривых Пеано, Дискрет. матем., 2013, том 25, выпуск 4, 66-73

DOI: https://doi.org/10.4213/dm1258

Использование Общероссийского математического портала Math-Net.Ru подразумевает, что вы прочитали и согласны с пользовательским соглашением http://www . mathnet.ru/rus/agreement

Параметры загрузки:

IP : 54.196 .121 .252

26 апреля 2023 г., 16:12:05 


\title{
Оценка снизу квадратно-линейного отношения для правильных кривых Пеано
}

\author{
(c) 2013 г. K. Е. Бауман
}

\begin{abstract}
В статье доказано, что квадратно-линейное отношение произвольной правильной кривой Пеано, отображающей единичный отрезок на единичный квадрат, не может быть менее пяти.

Работа выполнена при поддержке программы Отделения математики РАН (проект "Математика для вычислительных систем сверхвысокой производительности") и РФФИ (грант № 11-01-00822).
\end{abstract}

\section{1. Введение}

Правильной кривой Пеано, согласно [1], называется отображение отрезка на квадрат, допускающее разбиение области определения на несколько равных отрезков (фрактальных периодов) так, что ограничение кривой на любой из ее фрактальных периодов подобно всей кривой. Части кривой, соответствующие фрактальным периодам, называются ее фракииями. Минимальное число изометричных фракций, на которое разбивается кривая, называется ее фрактальным родом.

Чтобы применять кривые Пеано, нужно знать некоторые их свойства. Например, в приложениях, где требуется обход (сканирование) многомерной решетки, обычно необходимо знать, насколько далеко кривая уходит от заданной точки за определенное время. В разных случаях для различных кривых применялись различные способы это измерить $([6],[7],[8])$. Одним из способов является так называемое квадратно-линейное отношение, которое для пары $p(t), p(\tau)$ точек кривой Пеано $p:[0,1] \rightarrow[0,1] \times[0,1]$ определяется формулой

$$
\frac{|p(t)-p(\tau)|^{2}}{|t-\tau|} \text {. }
$$

Верхняя грань квадратно-линейных отношений для всевозможных пар различных точек кривой называется квадратно-линейным отношением кривой.

Для правильных фрактальных кривых Пеано разность параметров $|t-\tau|$ пропорциональна площади образа отрезка $[t, \tau]$. Для кривых, отображающих единичный отрезок на единичный квадрат (квадратных кривых), этот коэффициент пропорциональности равен единице, т. е. такие кривые параметризованы площадъю. Причем это свойство сохраняется и для их фракций. Поэтому в дальнейшем при оценивании квадратно-линейных отношений мы считаем все рассматриваемые кривые параметризованными площадью. 
В статье [2] доказано, что квадратно-линейное отношение кривой ПеаноГильберта равно шести. В статьях [3] и [5] приведены множество кривых Пеано с наименьшим квадратно-линейным отношением в классе правильных кривых Пеано фрактального рода 9, а также основанное на компьютерных вычислениях доказательство того, что это отношение равно $5 \frac{2}{3}$.

В статье [6] для широкого класса кривых, включающего правильные фрактальные кривые Пеано, доказано, что максимум квадратно-линейного отношения не менее 4.

Теорема 3 из [1] ошибочно утверждает, что начало и конец любой правильной фрактальной квадратной кривой Пеано совпадают с вершинами квадрата-образа. Приведенное там доказательство проходит только в тех случаях, когда при построении второго шага кривой не используется операция обращения времени.

На рис. 1 изображен контрпример к теореме 3, т. е. обход второго шага построения кривой фрактального рода 16, которая имеет свое начало в вершине квадрата, а конец - в середине стороны.

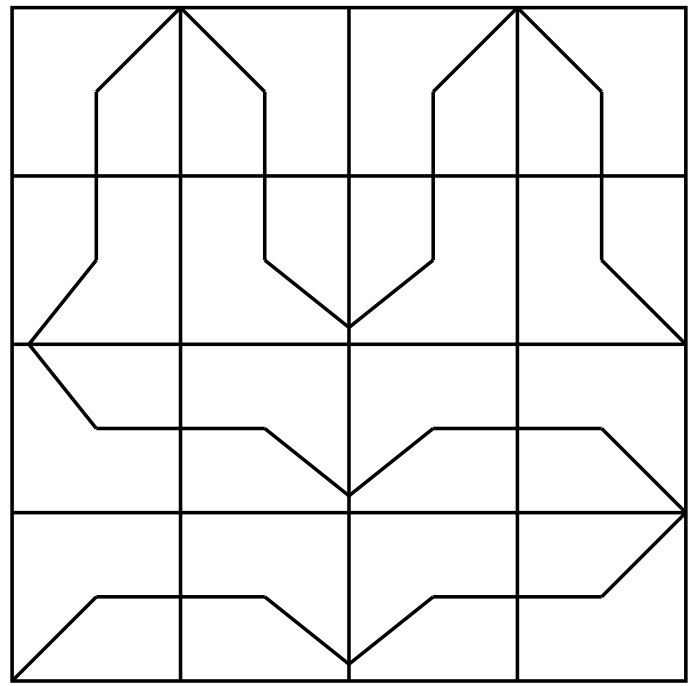

Рис. 1. Контрпример к теореме 3 из [1]

Теоремы 4 и 5 из статьи [1] утверждают, что квадратно-линейное отношение правильных фрактальных кривых Пеано, имеющих начало и конец в вершинах квадрата, не может быть меньше 5.

В настоящей статье доказана теорема, дополняющая статью [1] и позволяющая обосновать оценку снизу, равную пяти для всех правильных квадратных кривых Пеано.

Структура статьи следующая: в п. 2 доказана теорема о расположении начала и конца у любой правильной квадратной кривой Пеано, п. 3 посвящен оценке снизу квадратно-линейного отношения кривых с началом в углу квадрата, а концом в середине противоположной стороны. И наконец, в п. 2 формулируется и обосновывается основная теорема. 


\section{2. Расположение концов правильных кривых Пеано}

Лемма 1. У любой правильной кривой Пеано хотя бы одна из начальной и конечной точек лежит в вериине квадрата.

Доказательство. (Аналогичные рассуждения использовались в статье Щепина [1] в доказательстве теоремы 3.)

Допустим, что ни начало, ни конец кривой не лежат в вершинах квадрата. А значит, они могут лежать либо на одной стороне (1), либо на соседних сторонах квадрата (2), либо на противоположных сторонах квадрата (3).

Случай (1) невозможен, потому что в первой по обходу фракции второго шага построения вход и выход будут находиться на границе квадрата, не имеющей пересечения с другими фракциями.

Случай (3) также невозможен, потому что обход кривой на втором шаге построения не сможет сделать поворот, а значит, не будет возможности обойти все фракции.

Рассмотрим случай (2). Допустим, что кривая имеет начало и конец на соседних сторонах; тогда пересечение фракций с номерами, по обходу отличающимися на 2 , не пусто. Рассмотрим угловую фракцию на третьем шаге построения, которая от начала и от конца кривой удалена более, чем на 2 фракции. В таком случае она должна иметь непустое пересечение с фракциями, номера которых по обходу отличаются от ее номера не более, чем на 2 . Таких фракций 4 , а соседей у рассматриваемой угловой фракции всего 3. Противоречие.

Теорема 1. У любой правильной кривой Пеано начальная и конечная точки находятся либо в вершинах квадрата, либо одна из них в вершине, а другая в середине противоположной стороны.

Доказательство. По лемме 1 хотя бы одна из начальной и конечной точек лежит в вершине квадрата. Пусть начальная точка лежит в левой нижней вершине квадрата, в противном случае будем рассматривать кривую после обращения времени и соответствующего поворота.

Допустим, что конечная точка не лежит ни в вершине, ни в середине стороны квадрата.

Случай, когда конец лежит на одной стороне с началом, невозможен, потому что в противном случае в первой фракции вход и выход находились бы на границе квадрата, не имеющей пересечения с другими фракциями.

Пусть конец лежит на противоположной стороне от начала, например, на правой вертикальной стороне квадрата, и не попадает в середину, т.е. находится на расстоянии $x$ от нижней границы квадрата, причем $x \notin\left\{0, \frac{1}{2}, 1\right\}$. В таком случае на втором шаге построения после любой фракции, ориентированной так же, как и вся кривая, может идти только фракция, зеркально отраженная к данной относительно их общей границы. Так как первая фракция, как и вся кривая, имеет начало в вершине, значит, конец второй фракции также попадает в вершину разбиения. А значит, все нечетные по обходу фракции имеют начало в вершине разбиения, а у четных в вершину попадает конец.

Введем координаты с началом в левом нижнем углу квадрата, оси координат направим вдоль сторон квадрата, а за единицу примем длину стороны фракции второго шага построения кривой. 
Заметим, что тогда координаты конца второй фракции могут иметь вид либо $(2,0)$, либо $(0,2)$, а координаты любого конца или начала фракции, попавшего в вершину разбиения, являются четными числами.

Допустим, что кривая имеет четный фрактальный род; тогда левая верхняя фракция имеет свое начало или конец в левой верхней вершине квадрата. Она не может являться концом всей кривой, но и двух соседей по обходу она также иметь не может. Противоречие.

Допустим, что кривая имеет нечетный фрактальный род; тогда правая верхняя фракция начинается в своем левом нижнем углу, а заканчивается на стороне большого квадрата, не имеющей пересечения с остальными фракциями. А значит, такая фракция может быть только конечной. Но в таком случае отображение подобия всей кривой на эту фракцию оставляет единственную неподвижную точку в углу квадрата, следовательно, конец кривой будет лежать в правом верхнем углу квадрата, что противоречит изначальному предположению.

\section{3. Оценка снизу для специальных кривых}

Пятеричные кривые. Согласно статье [1], мы будем называть правильную квадратную пеановскую кривую пятеричной, если она подобна единичной пеановской кривой и имеет квадратно-линейное отношение менее 5 .

Обозначим буквой $K$ класс правильных квадратных пеановских кривых, имеющих свое начало в вершине квадрата, а конец в середине противоположной стороны. Все доказательства мы будем проводить для класса $K$, имея в виду, что для кривых с началом в середине стороны и концом в вершине квадрата они идентичны с точностью до обращения времени.

Теорема 2. Класс кривых $K$ не содержит пятеричных кривых.

Доказательство теоремы основывается на серии лемм.

Лемма 2. У любой кривой из $K$ любъе две соседние фракиии имеют противоположные ориентации по времени.

Доказательство. Так как понятие правильной пеановской кривой подразумевает правильное разбиение квадрата, то в ней невозможен стык между вершиной квадрата и серединой стороны соседнего квадрата. А значит, две соседние по обходу фракции кривой из класса $K$ не могут иметь совпадающую ориентацию по времени.

Рассмотрим стык двух фракций, где первая изначальна (т.е. ее ориентация по времени совпадает с ориентацией всей кривой [1]), а вторая (по лемме 2) имеет обратную ориентацию по времени. Поворотами и отражениями всей кривой можно добиться того, чтобы первая фракция начиналась в ее левом нижнем углу, а заканчивалась в середине ее правой стороны. Тогда вторая фракция имеет свое начало в середине своей левой стороны, а конец в одной из вершин правой стороны. Если ее конец лежит в правой нижней вершине, то такой стык назовем зеркальным, а если в правой верхней вершине, то стыком с переворотом.

Лемма 3. У любой кривой из класса $K$ найдется стык с переворотом. 
Доказательство. Пусть начальная точка лежит в левой нижней вершине квадрата, а конец - в середине правой стороны; в противном случае будем рассматривать кривую после соответствующего поворота.

Введем координаты с началом в левом нижнем углу квадрата, оси направим вдоль сторон квадрата, а за единицу примем длину стороны фракции второго шага построения кривой.

Допустим, что у кривой встречаются только зеркальные стыки.

Заметим, что координатами конца второй фракции могут быть либо $(2,0)$, либо $(0,2)$, а также, что координаты любого конца или начала фракции, попавшего в вершину разбиения, являются четными числами.

Допустим, что кривая имеет четный фрактальный род; тогда левая верхняя фракция имеет свое начало или конец в левой верхней вершине квадрата. Она не может являться концом всей кривой, но и двух соседей по обходу она также иметь не может, значит, обход с такими условиями невозможен.

Допустим, что кривая имеет нечетный фрактальный род; тогда правая верхняя фракция начинается в своем левом нижнем углу, а заканчивается на стороне большого квадрата, не имеющей пересечения с остальными фракциями, но конечной эта фракция быть не может по условию кривых класса $K$. Значит, такой обход также невозможен.

Итак, вне зависимости от четности фрактального рода кривой доказана невозможность ее построения с помощью только зеркальных стыков.

Лемма 4. Пятеричные кривые из класса $K$ не содержат диагоналъных стыков.

Доказательство. Допустим, что у пятеричной кривой из класса $K$ есть диагональный стык.

Рассмотрим варианты взаимного расположения фракций следующего шага при диагональном стыке (рис. 2). Первая и вторая фракции от угла всегда граничат по стороне, и так как начало первой фракции в вершине, то переход во вторую осуществляется в середине стороны.
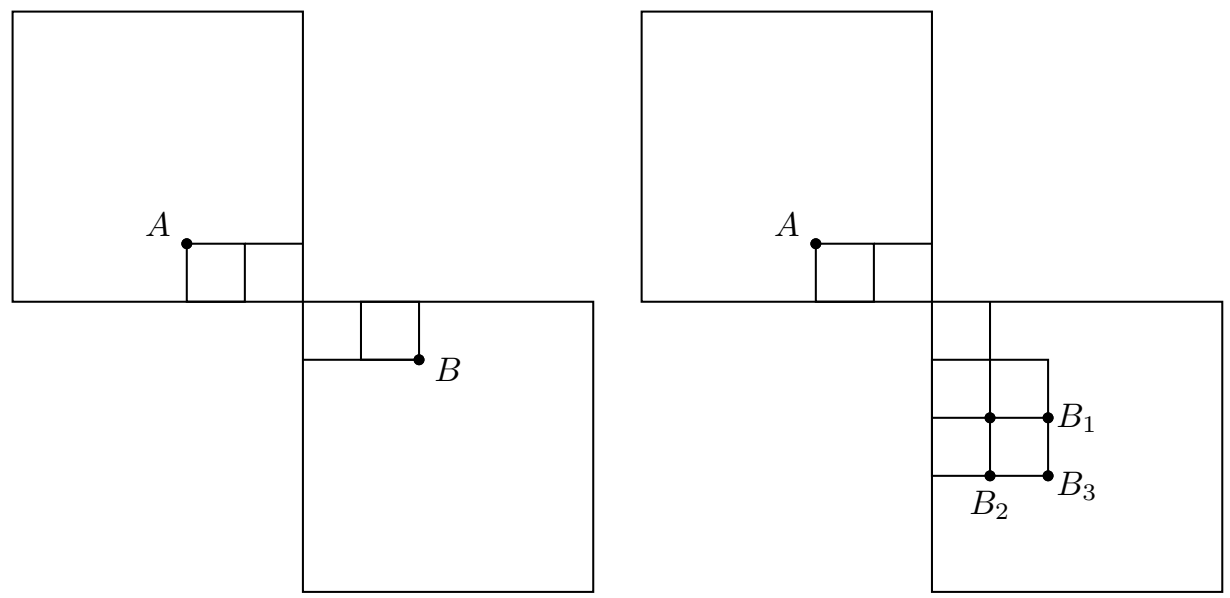

Рис. 2. Диагональный стык

В одном случае (рис. 2 слева) достаточно рассмотреть по паре фракций следующего шага от вершины стыка и вычислить нижнюю оценку квадратно-линейного 
отношения между отмеченными точками:

$$
F(A, B) \geqslant \frac{2^{2}+4^{2}}{4}=5
$$

Во втором случае (рис. 2 справа) заметим, что переход между второй и третьей фракциями от угла происходит в вершине, а значит, существуют три фракции, которые могут быть третьими по обходу от угла. Во всех трех случаях рассмотрим дальнюю вершину третьей фракции от вершины диагонального стыка. На рис. 2 (правый) эти точки обозначены $B_{1}, B_{2}, B_{3}$. Вычислим нижнюю оценку квадратнолинейного отношения на отмеченных точках:

$$
F\left(A, B_{i}\right) \geqslant \frac{3^{2}+4^{2}}{5}=5 .
$$

В обоих случаях приходим к противоречию с пятеричностью кривой.

Лемма 5. В классе $K$ нет пятеричных кривых четного фрактального рода.

Доказательство. Рассмотрим кривую четного фрактального рода из класса $K$. Так как по лемме 2 ориентации фракций второго шага построения чередуются, а первая фракция изначальна, то последняя фракция имеет противоположную ориентацию по времени, то есть выход кривой из последней фракции происходит в ее вершине. Вспомним, что по лемме 3 у кривой найдется стык с переворотом, а значит, в этом стыке на следующем шаге построения кривой возникнет диагональный стык. Такая кривая не может быть пятеричной по лемме 4.

Лемма 6. В классе $K$ нет пятеричных кривых нечетного фракталъного рода.

Доказательство. Предположим противное: в классе $K$ существует хотя бы одна пятеричная кривая нечетного фрактального рода.

Так как по лемме 2 ориентации фракций второго шага построения чередуются, а первая фракция изначальна, то последняя фракция также изначальна, то есть выход кривой из последней фракции происходит в середине ее стороны.

Вспомним, что по лемме 3 у кривой найдется стык с переворотом, и рассмотрим его подробнее. На рис. 3 изображен стык, у которого левая фракция начинается в левом нижнем углу, правая заканчивается в правом верхнем углу, переход происходит в середине их общей стороны. А также на рисунке изображены некоторые фракции следующего шага построения кривой. Для удобства объяснения мелкие фракции занумерованы.

Фракции с номерами 0 являются соответственно последней в левой фракции и первой в правой фракции, переход между ними происходит в середине их общей стороны. Так как фрактальный род кривой нечетен, значит, первая и последняя фракция кривой изначальны, а значит, у фракции с номерами 0 переходы к соседям происходят в вершинах разбиения.

Будем рассматривать обход кривой в правой фракции, так как в левой он симметричен.

Пусть выход из фракции с номером 0 осуществляется в правом нижнем углу, иначе будем проводить доказательство после отражения стыка относительно горизонтали. 


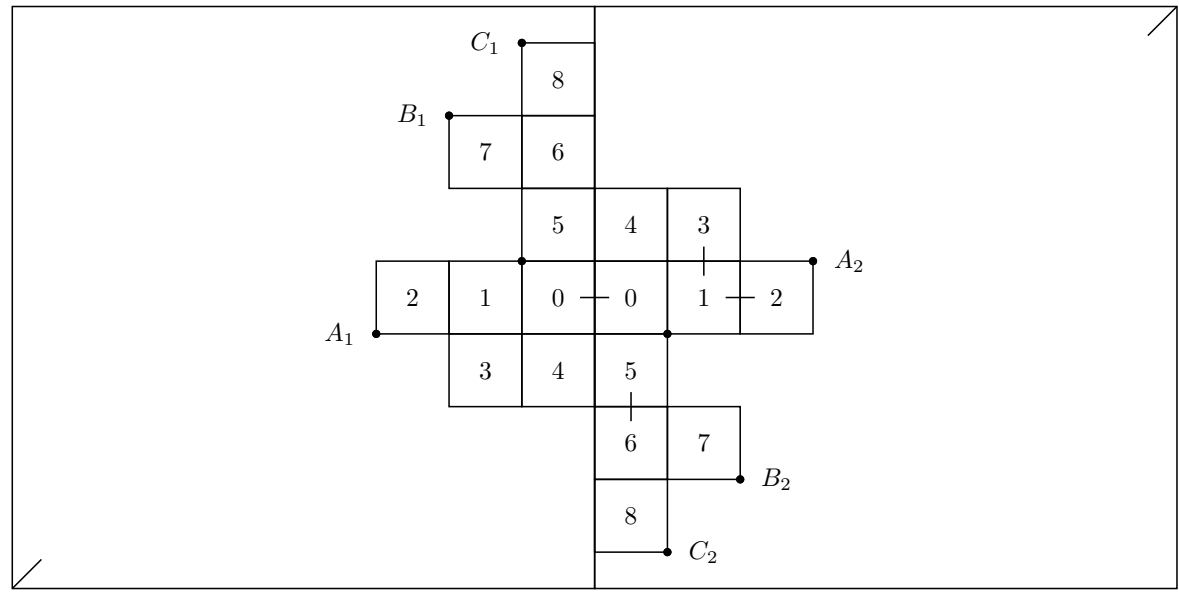

Рис. 3. Стык с переворотом у кривой с нечетным фрактальным родом

Напомним, что: 1) по предположению кривая пятеричная, значит, по лемме 4 она не имеет диагональных стыков; 2) по лемме 2 стык между второй и третьей по обходу фракциями происходит в середине стороны. А значит, возможны следующие последовательности посещения подфракций правой фракции стыка: $(0,1,2),(0,1,3),(0,5,6,7),(0,5,6,8)$.

В случае $(0,1,2)$ вычислим оценку

$$
F\left(A_{1}, A_{2}\right) \geqslant \frac{6^{2}+1}{6}>5
$$

Она противоречит пятеричности.

В случае $(0,1,3)$ заметим, что фракцию под номером 4 нельзя обойти, значит, такой обход невозможен.

Для случаев $(0,5,6,7)$ и $(0,5,6,8)$ вычислим нижнюю оценку квадратнолинейного отношения для соответствующих пар точек:

$$
F\left(B_{1}, B_{2}\right) \geqslant \frac{4^{2}+5^{2}}{8}>5 ; F\left(C_{1}, C_{2}\right) \geqslant \frac{7^{2}+2^{2}}{8}>5 .
$$

Таким образом, рассмотрены все обходы, удовлетворяющие предположению, и доказано, что в любом случае у кривой из класса $K$ нечетного фрактального рода квадратно-линейное отношение не менее 5.

Доказательство (теоремы 2). Класс кривых $K$ состоит из кривых четного и нечетного фрактальных родов. Но по леммам 5 и 6 ни те, ни другие не содержат пятеричных кривых.

\section{4. Оценка снизу квадратно-линейного отношения правильных кривых Пеано}

Теорема 1 утверждает, что у любой правильной пеановской кривой начальная и конечная точки находятся либо в вершинах квадрата, либо одна из них в вершине, 
а другая - в середине противоположной стороны. Теоремы 4 и 5 из [1] утверждают, что у правильных кривых Пеано с началом и концом в вершинах квадрата квадратно-линейное отношение не менее 5 .

Таким образом, теорема 2 дополняет статью [1] и обосновывает следующее утверждение.

Теорема 3. Всякая правильная квадратная кривая Пеано имеет квадратнолинейное отношение не менее 5 .

\section{Список литературы}

1. Щепин Е. В., О фрактальных кривых Пеано. Трудъ МИАН, (2004) 247, 204-303.

2. Бауман К. Е., Коэффициент растяжения кривой Пеано-Гильберта. Матем. заметки (2006) 80, №5, 643-656.

3. Бауман К. Е., Односторонние кривые Пеано фрактального рода 9. Труды МИАН (2011) 275, 55-67.

4. Щепин Е. В., Бауман К. Е., Минимальная кривая Пеано, Геометрия, топология и математическая физика. I, Сборник статей. K 70-летию со дня рождения академика С. П. Новикова,. Труды МИАН (2008) 263, 251-271.

5. Щепин Е. В., Бауман К. Е., О кривых Пеано фрактального рода 9. Моделирование $u$ анализ данных: Труды факультета информационных технологий МГППУ (2004), № 1 79-89.

6. Haverkort H., Walderveen F., Locality and bounding-box quality of two-dimensional spacefilling curves. arXiv:0806.4787v2 [cs.CG].

7. Niedermeier R., Reinhardt K., Sanders P., Towards optimal locality in mesh-indexings. Discrete Appl. Math. (2002) 117, №1-3, 211-237.

8. Gotsman C., Lindenbaum M., On the tetric properties of discrete space-filling curves. IEEE Trans. Image Proc. (1996) 5, №5,. 